\title{
Auslegung hybrider Querpressverbände für erhöhte Betriebstemperaturen
}

\author{
Miriam Wagner ${ }^{1}$ (D) $\cdot$ Hansgeorg Binz ${ }^{1}$ \\ Eingegangen: 26. September 2019 / Angenommen: 27. November 2020 / Online publiziert: 11. Dezember 2020 \\ ○ Der/die Autor(en) 2020
}

\section{Zusammenfassung}

Die vorliegende Veröffentlichung befasst sich mit der Auslegung hybrider Querpressverbände, d.h. der Verbindung von Stahlwellen und vollkeramischen Naben, bei Betriebstemperaturen oberhalb der Raumtemperatur. Zunächst wird in einer Einführung auf die Vorteile dieser Verbindung eingegangen. Es folgt ein Überblick über den Stand der Forschung und die erzielten Ergebnisse bei Raumtemperatur. Die zwei bisher vorhandenen Auslegungsverfahren bedienen sich strukturmechanischer bzw. thermomechanischer Simulationen, um die wirkenden Kräfte auf die Fügepartner und die daraus resultierenden Verformungen zu berechnen. Mit den Ergebnissen wird eine neue Wellenform definiert. So gefertigte Wellen führen im Pressverband zu einem annähernd konstanten Fugendruckverlauf.

Simulationen zeigen jedoch, dass sich diese Wellen nicht für den Betrieb bei erhöhten Temperaturen eignen. Um einen ähnlich günstigen Spannungszustand zu erreichen und Spannungsspitzen zu vermeiden, muss das Vorgehen modifiziert werden. Die hierfür notwendigen Schritte werden ausführlich beschrieben. Darüber hinaus werden anwendungsnahe Hinweise zur Optimierung der Ergebnisse bzw. geometrischen Gestaltung des Pressverbands gegeben.

\section{Design of hybrid interference fits for increased operating temperatures}

\begin{abstract}
This paper describes the dimensioning of hybrid interference fits for operating temperatures higher than room temperature. Hybrid interference fits are understood as combination of ceramic hubs and metal shafts. First of all, the benefits of such a connection are explained. Afterwards, an overview of the state of research and results gained for room temperature is given. The two existing methods use structural respectively thermomechanical simulations to calculate the occuring forces on shaft and hub as well as the resulting deformations. The output is utilized to define a new shaft geometry. Shafts manufactured this way lead to an almost constant pressure level on the inside of the interference fit.

However, simulations show that these shafts are not suitable for operation at increased temperatures. The method has to be modified in order to achieve an adequate stress distribution and to avoid stress peaks. All necessary steps for this purpose are shown explicitly. Furthermore, application-related suggestions are given for optimization of results respectively geometrical designing of the interference fit.
\end{abstract}

\section{Einleitung}

Miriam Wagner

miriam.wagner@iktd.uni-stuttgart.de

Hansgeorg Binz

hansgeorg.binz@iktd.uni-stuttgart.de

1 Institut für Konstruktionstechnik und Technisches Design, Universität Stuttgart, Pfaffenwaldring 9, 70569 Stuttgart,

Deutschland
Pressverbände als Welle-Nabe-Verbindungen zählen zu den häufigsten Maschinenelementen im Maschinenbau. Sie übertragen das eingeleitete Drehmoment reibschlüssig über eine zylindrische Fügefläche. Die benötigte Flächenpressung wird durch ein entsprechendes Übermaß zwischen Welle und Nabe erreicht. Dank ihrer einfachen Grundgeometrie sind sie relativ kostengünstig in der Herstellung. Gleichzeitig reagieren sie sehr tolerant auf kurzzeitige Überlast. Wo die Auswahl an metallischen Naben z.B. 
durch extreme Temperaturen oder korrosive Einflüsse anwendungsbedingt stark eingeschränkt oder deren Einsatz gar nicht mehr möglich ist, können keramische Werkstoffe eine zweckmäßige Option sein.

Keramiken sind gegenüber chemischen und abrasiven Einflüssen extrem beständig und zeichnen sich durch ihre große Härte aus. Nachteilig kann sich ihre Sprödigkeit und damit einhergehende Empfindlichkeit bezüglich Zugspannungen auswirken. Anders als metallische Materialien können Keramiken nicht fließen. In Folge zu hoher Belastung reagieren sie deshalb meistens mit sofortigem Sprödbruch. Zugspannungen müssen also konstruktiv vermieden bzw. im (sicher) ertragbaren Bereich gehalten werden.

Nachdem im Pressverband prinzipbedingt Zugspannungen in der Nabe auftreten, gab es in der Vergangenheit zunächst andere Ansätze zur Anbindung der keramischen Bauteile. Unter den reibschlüssigen Varianten gehörten dazu insbesondere Versuche mit Keramikwellen, die im Fügebereich von Stahlnaben umschlossen werden [1]. Teilweise wurden diese mit Zwischenstücken aus Bimetall ausgeführt [2]. In allen Fällen gab es konstruktive Schwierigkeiten oder war das übertragbare Drehmoment stark begrenzt. Durch die geringere Wärmeausdehnung der meisten keramischen Werkstoffe im Vergleich zum Stahlpartner kann es bei innenliegender Keramik zu einem Verlust der Pressung kommen, wenn die Bauteiltemperatur steigt.

Der hybride Querpressverband, wie er von Blacha [3] definierte wurde, bezeichnet einen Pressverband bestehend aus einer vollkeramischen Nabe und einer Stahlwelle. Wie bei Querpressverbänden gängig, wird die Welle mit definiertem Übermaß gefertigt und beide Teile werden über eine Temperaturdifferenz gefügt. Durch geeignetes Erwärmen oder Abkühlen eines oder beider Fügepartner dehnen diese sich aus bzw. ziehen sich zusammen, sodass ein Fügespiel entsteht und sie ineinander positioniert werden können. Mit dem anschließenden Temperaturausgleich entsteht eine starke radiale Pressung, über die Drehmomente übertragen werden können. Gleichzeitig entstehen in der Nabe Zugspannungen, die in zu großer Höhe zum Versagen der Nabe führen können. Die Auslegung nach Blacha [3] ermöglicht die sichere und dauerfeste Anbindung einer Keramiknabe an eine Stahlwelle. Durch eine gezielte Modifizierung des Wellendurchmessers über der Wellenlänge werden die auftretenden Zugspannungen in der Nabe im zulässigen Rahmen gehalten. Die notwendigen Anpassungen des Durchmessers werden im Rahmen einer sogenannten Fugendruckhomogenisierung berechnet, ein von Blacha [3] entwickeltes Verfahren, das zu einer gleichmäßig hohen Pressung im gesamten Pressverband führt. In dieser Konstellation kann es in Abhängigkeit von den materialspezifischen und geometrischen Verhältnissen bei Temperaturerhöhung zu einem Spannungsanstieg kommen. Voruntersuchungen haben gezeigt, dass Blachas für Raumtemperatur berechnete Wellenform nicht bei davon abweichenden (in der Regel höheren) Betriebstemperaturen angewendet werden kann. Im Folgenden wird deshalb ein anderer Ansatz zur Auslegung vorgestellt.

Eingesetzt werden kann dieser hybride Querpressverband insbesondere in Förder- bzw. Pumpenanlagen zur Anbindung von Pumpenlaufrädern. Durch die hohe Härte der Keramik können abrasive Stoffe transportiert werden und durch ihre weitgehende chemische Inertheit sind chemisch agressive Medien ebenfalls ein Anwendungsgebiet. Dabei sind gleichzeitig auftretende hohe Temperaturen keine Einschränkung. Die hohe Temperaturbeständigkeit kann außerdem in Turboladern genutzt werden.

\section{Stand der Forschung}

Blacha definierte bereits 2009 eine erste Richtlinie zur Gestaltung der Fügefläche zwischen Stahlwellen und vollkeramischen Naben [3], mit deren Hilfe sich die Pressverbindung dauerfest auslegen lässt. Unter Einsatz aufwändiger Studien und Voruntersuchungen entwickelte er ein einfach anwendbares Verfahren auf Basis rein strukturmechanischer Simulationen. Diesem liegt die Tatsache zugrunde, dass durch den Pressverband sowohl Welle als auch Nabe in der Fügefläche vordergründig durch eine radiale Pressung und durch axiale Reibkräfte belastet werden. Die Reibkräfte entstehen durch den Fügevorgang. Für diesen wird in der Regel die Welle abgekühlt und/oder die Nabe erwärmt. Die Temperaturdifferenz bewirkt ein Fügespiel, durch das beide Teile ineinander positioniert werden können. Während des folgenden Temperaturausgleichs kommt es zum Aufbau der Pressung (häufig auch als Fugendruck bezeichnet). Gleichzeitig findet durch die temperaturbedingte Dehnungsänderung eine entgegengesetzte axiale Bewegung zwischen den Körpern statt, die sich im Kontakt in Form von Reibkräften auswirkt. Wird nun einer der Fügepartner getrennt betrachtet, so wirken auf seine Fügefläche der radiale Fugendruck und die axialen Reibkräfte. Diese Lasten verformen den Körper.

Ziel der Auslegung ist ein konstanter Fugendruck über der gesamten Fügelänge. Dazu werden in einem ersten Schritt getrennte Modelle von Welle und Nabe jeweils mit dem gewünschten Fugendruck $p$ und Reibkräften $\mathrm{F}_{\text {reib }}$ beaufschlagt (siehe Abb. 1). Der Fugendruck kann aus der maximal zulässigen Zugspannung in der Keramik berechnet werden. Hierfür werden die gängigen Formeln nach DIN 7190 [4] für Pressverbände angewandt. Höhe und Verteilung der Reibkräfte sind am Anfang unbekannt. Blacha fand auf Basis von Überlegungen und Vergleichssimulationen in einer Studie heraus, dass der von ihm als Dreiecksmodell bezeichnete Verlauf am geeignetsten ist, um die Reibkräfte zu modellieren. In einem iterativen Vorgehen 

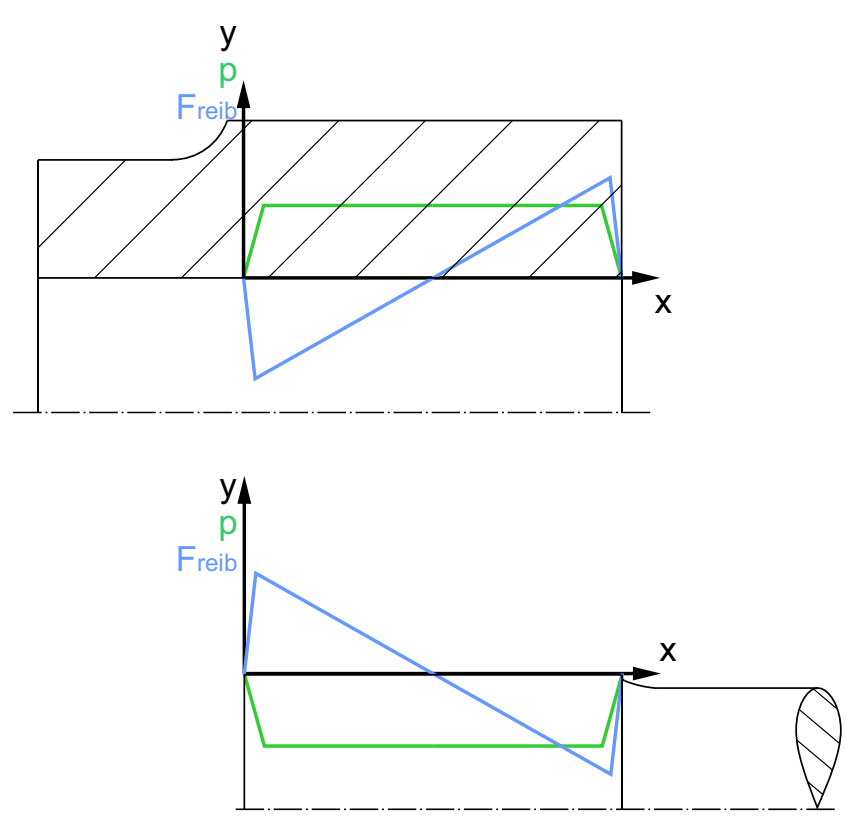

Abb. 1 Prinzip der aufgebrachten Kräfte zur Ermittlung der Wellenkontur nach [3]

muss die korrekte Höhe „des Dreiecks“ bzw. der Reibkräfte für die jeweilige Geometrie und zulässige Spannung gefunden werden. Im nächsten Schritt werden die getrennt ermittelten radialen Verschiebungen $\Delta \mathrm{y}$ von Welle und Nabe addiert (siehe Abb. 2). Daraus entsteht die sogenannte Kontur. Wird nun eine Welle mit dieser Kontur gefertigt und mit einer rein zylindrischen Nabe gefügt, so ergibt sich im gefügten Zustand der gewünschte konstante Fugendruck.

Nachdem die Reibsimulationskräfte von Blacha durch die Iteration aufwändig zu bestimmen sind und dennoch nur eine Annäherung bleiben, wurde dieser Teil der Berechnung 2015 von Krautter [5] verändert. Dazu wurde eine thermomechanische Simulation des Fügevorgangs im-

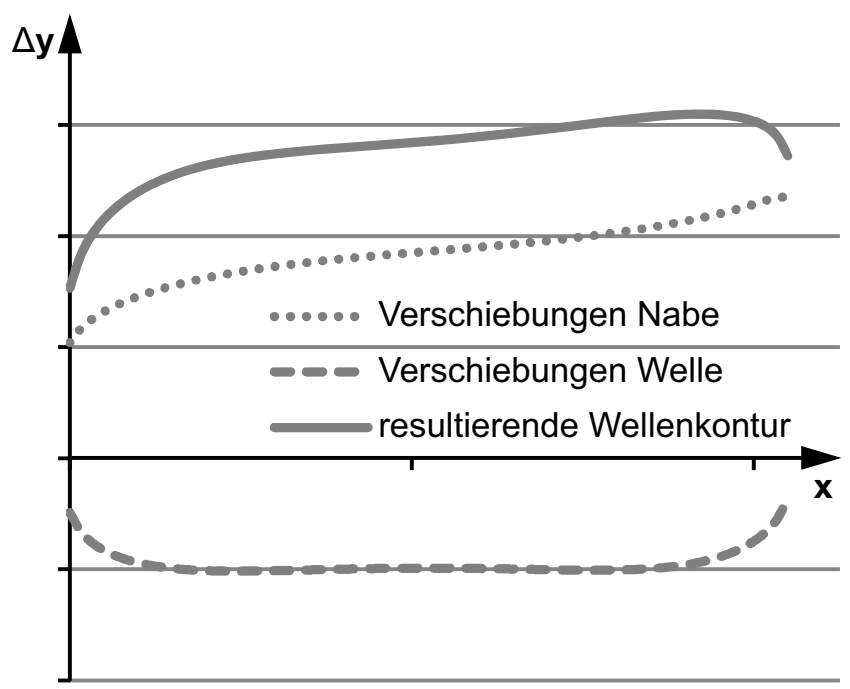

Abb. 2 Addition der Verschiebungen zur Wellenkontur nach [3] plementiert ab dem Moment, in dem Welle und Nabe zueinander positioniert sind und der Temperaturausgleich beginnt. Zunächst wird von einem konstanten Übermaß über der Fügelänge ausgegangen. Dieses Übermaß lässt sich entsprechend der Nabengeometrie (insbesondere des Durchmesserverhältnisses) nach DIN 7190 aus dem zulässigen Fugendruck bzw. der zulässigen Zugspannung berechnen. Ausgewertet wird anschließend der Reibkraftverlauf über der Zeit.

Die Reibkräfte starten bei null, denn durch das Fügespiel besteht noch ein geringer Abstand zwischen Welle und Nabe. Durch den Wärmeaustausch mit der Umgebung bzw. dem Fügepartner kommt es bereits nach sehr kurzer Zeit zu einer Berührung. Diese muss in Abhängigkeit von den geometrischen Verhältnissen nicht sofort auf der ganzen Länge stattfinden. Indem sich die Welle ausdehnt und die Nabe zusammenzieht, entsteht eine Relativbewegung zwischen beiden, durch die auch axiale Reibkräfte entstehen. Je weiter der Temperaturangleich fortschreitet, desto größer werden die sich berührenden Bereiche und umso größer wird auch die Pressung, die sich in radialer Richtung ausbildet. So steigt die Summe der Reibkräfte auf ein Maximum an. An den Fügeoberflächen stellt sich durch den engen Kontakt zuerst dieselbe Temperatur ein. Nach und nach erreichen beide Teile Raumtemperatur und auch die Pressung erreicht ihren Wert im fertig gefügten Zustand. Abhängig von den Starttemperaturen und geometrischen Verhältnissen kann die Pressung gegen Ende des Fügens geringer sein als während des Vorgangs. In diesen Fällen sinken die Reibkräfte nach Erreichen des Maximums teilweise wieder ab.

Der Zeitpunkt, zu dem die Summe der Reibkräfte maximal ist, wird weiterverwendet (vgl. Abb. 3). Mit diesen Reibkräften und dem wieder konstanten Fugendruck wer-

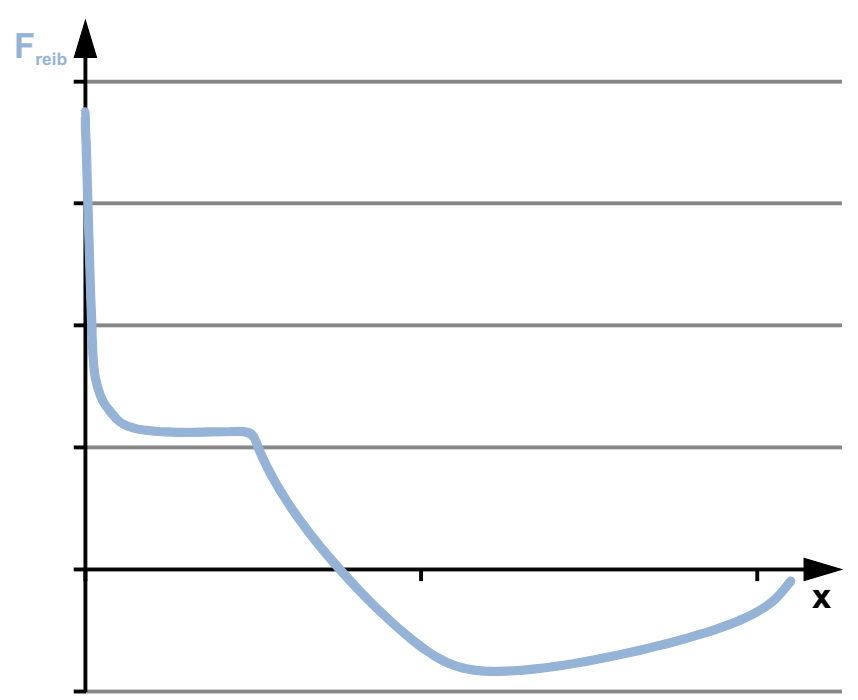

Abb. 3 Reibkraftverlauf für die Welle nach [5] 

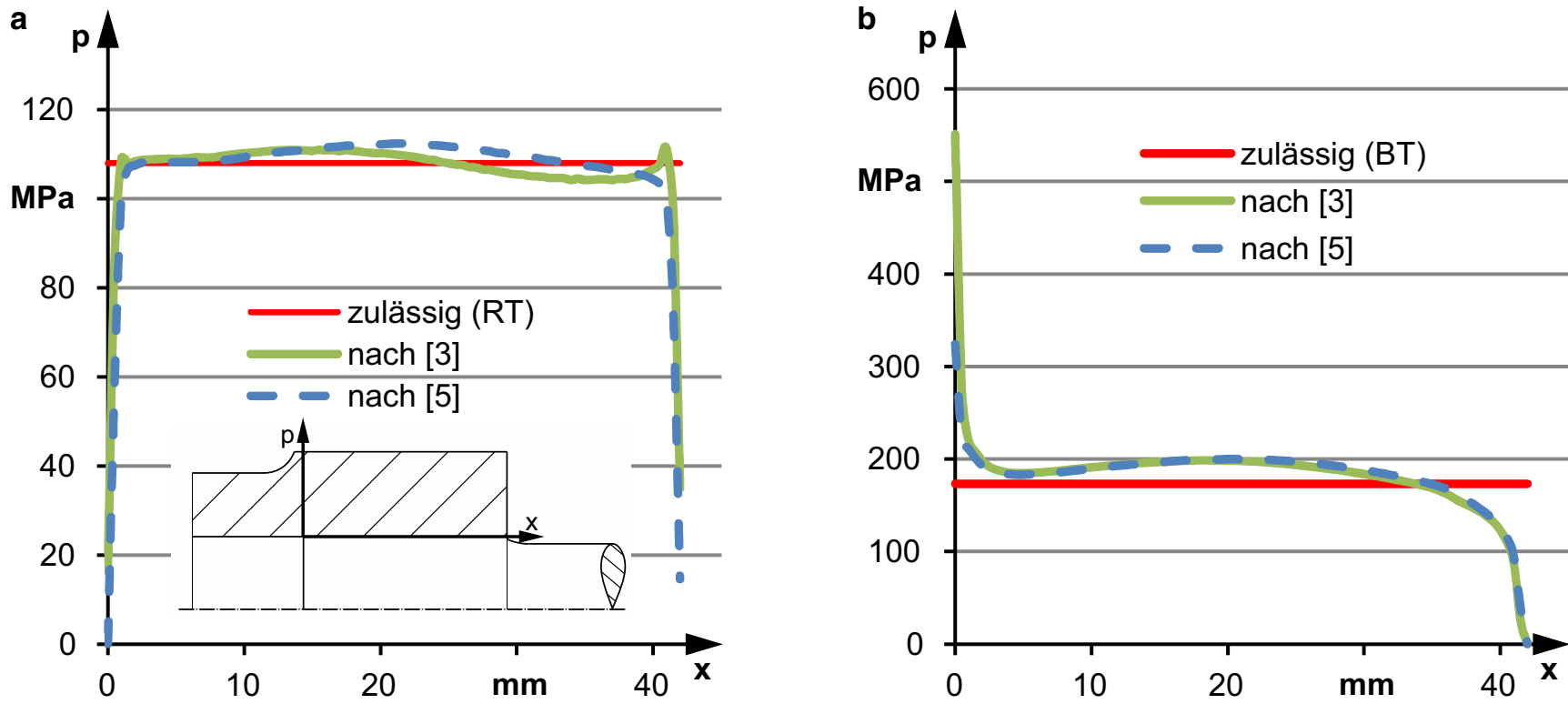

Abb. 4 Fugendruck bei Berechnung nach [3] und [5] bei RT $=20^{\circ} \mathrm{C}$ (a) und $\mathrm{BT}=90^{\circ} \mathrm{C}(\mathbf{b})$

den nun die getrennten Modelle von Welle und Nabe belastet. Die Berechnung der Kontur erfolgt analog zu Blacha.

\section{Auslegung für erhöhte Betriebstemperaturen}

Ein großer Vorteil von Keramiken besteht in ihrer großen Hitzebeständigkeit (oft weit über $1000^{\circ} \mathrm{C}$ ). In Verbindungen mit metallischen Bauteilen kann diese Eigenschaft nicht voll ausgereizt werden, weil es hier schon deutlich früher zu Gefügeveränderungen und damit einhergehend zu Veränderungen im Materialverhalten, insbesondere der Festigkeit kommt. Dennoch können auch Stähle erhöhte Temperaturen ertragen und so ist es naheliegend, für den hybriden Pressverband Betriebstemperaturen oberhalb der Raumtemperatur (RT, Annahme $20^{\circ} \mathrm{C}$ ) vorzusehen. Wie seitens der Auslegung mit solchen Anwendungsfällen umzugehen ist, wird im vorliegenden Beitrag untersucht.

Für sämtliche Simulationen wurden beispielhaft die Materialdaten von Siliziumnitrid $\left(\mathrm{Si}_{3} \mathrm{~N}_{4}\right)$ für die Nabe und Vergütungsstahl (42CrMo4) für die Welle zugrunde gelegt. Die Probengeometrie selbst wurde unverändert von Blacha [3] übernommen. Das Verhältnis von Nabeninnendurchmesser (Fügedurchmesser) zu Nabenaußendurchmesser $\mathrm{Q}_{\mathrm{A}}$ beträgt 0,46 bei einer Fügelänge von $42 \mathrm{~mm}$. Sowohl die strukturals auch die thermomechanischen Simulationen wurden mit zweidimensionalen achsensymmetrischen Modellen durchgeführt.

Einleitend wurden die bekannten Auslegungsverfahren betrachtet. Mittels thermomechanischen Simulationen kann gezeigt werden, dass die Konturen beider in Abschn. 2 ge- nannter Verfahren zwar bei Raumtemperatur gute Ergebnisse liefern (vgl. Abb. 4a), bei höheren Betriebstemperaturen (BT) jedoch zu unerwünschten Spannungsspitzen führen (vgl. Abb. 4b). Bei der Kontur nach [3] liegt die Spitze etwa 3,2-mal so hoch wie der zulässige Wert, bei der Kontur nach [5] immerhin noch beim 1,9-fachen Wert. In der Mitte des Pressverbands weicht der Fugendruck in beiden Fällen um etwa $15 \%$ nach oben ab. Diese Abweichungen, vor allem aber die hohen Spitzen im Randbereich, sind unbedingt zu minimieren, weil die spröde Keramik sie - anders als Stahl - nicht durch Plastifizieren abbauen kann. Das Überschreiten der zulässigen Spannung birgt deshalb im Allgemeinen immer die Gefahr eines spontanen Versagens durch Bruch. In einem solchen Fall wäre der Pressverband irreversibel zerstört und unbrauchbar.

Es stellt sich daher die Frage, wie der hybride Querpressverband für Betriebstemperaturen oberhalb der Raumtemperatur ausgelegt werden muss, um einen möglichst konstanten Fugendruck zu erreichen und das Auftreten von Spannungsspitzen zu vermeiden. Gleichzeitig soll der Ablauf des Verfahrens nicht komplizierter werden und damit weiterhin anwenderfreundlich bleiben.

Für konkrete Anwendungsfälle wird üblicherweise vom zu übertragenden Drehmoment ausgegangen. Gemäß [4] kann dazu das geforderte Moment in ein benötigtes Übermaß umgerechnet werden. Durch ihr sprödes Materialverhalten sowie die überwiegende Zugbelastung in der $\mathrm{Na}$ be, stellt die Keramik beim hybriden Querpressverband das kritischere Bauteil dar. Folglich muss geprüft werden, ob der Pressverband mit dem ermittelten Übermaß und der verfügbaren Keramik realisierbar ist. Ausgehend von der zulässigen Spannung der Keramik, die durch das Material 


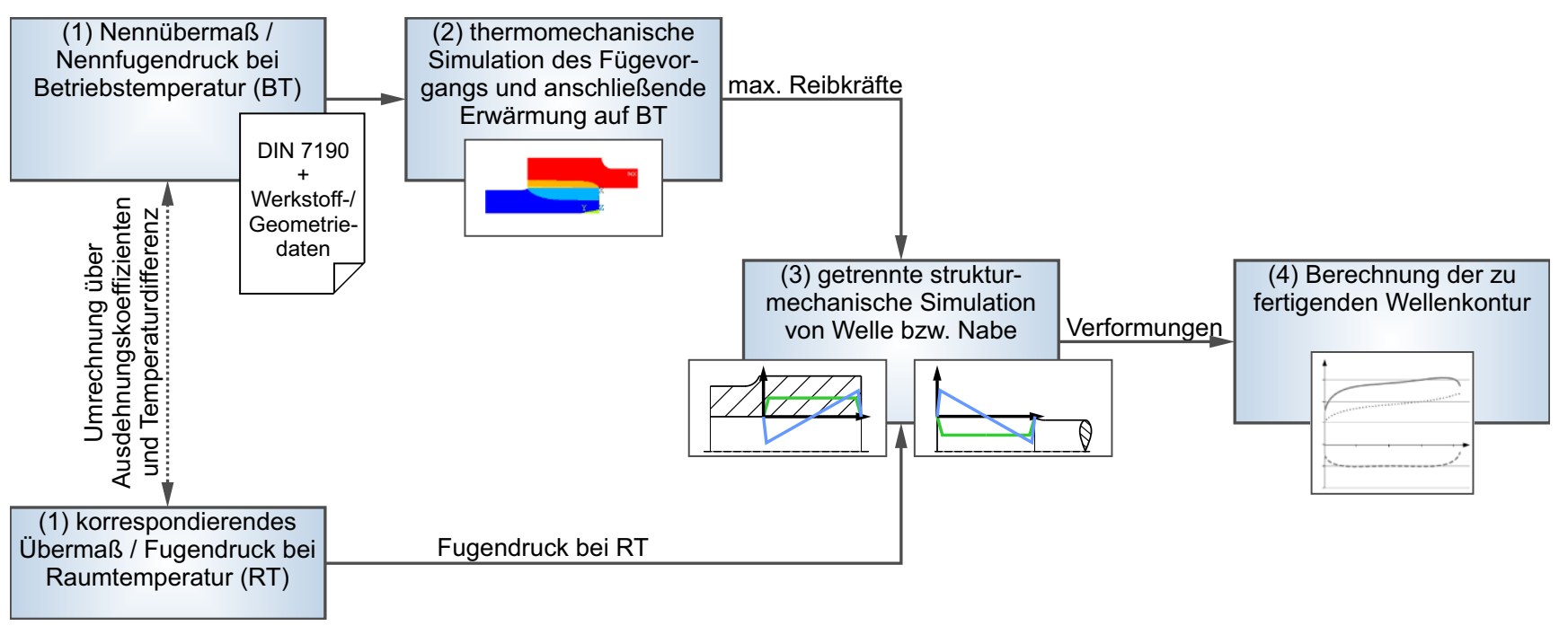

Abb. 5 Ablauf modifiziertes Verfahren

und das Bauteilvolumen bedingt ist [6], wird das mögliche Übermaß nach [4] berechnet. Liegt das benötigte Übermaß maximal auf Höhe des möglichen Übermaßes, so kann der Pressverband grundsätzlich gefertigt werden und die Feingestaltung kann beginnen. Das benötigte Übermaß wird dann als Nennübermaß des Pressverbands bezeichnet. Gilt dies nicht, müssen andere Werkstoffe in Betracht gezogen oder Sicherheitsfaktoren überprüft werden.

Maßgeblich für den sich ausbildenden Spannungszustand in der Keramiknabe sind vor allem zwei Faktoren. Zum einen haben die allermeisten Keramiken niedrigere Wärmeausdehnungskoeffizienten als Stahl. Das bedeutet, dass sich die Werkstoffe bei gleicher Temperaturdifferenz unterschiedlich stark ausdehnen. Im Fall des hybriden Pressverbands mit innenliegender Stahlwelle führt eine Temperaturerhöhung also zwangsläufig zu einer generellen Erhöhung des Spannungsniveaus in der Keramiknabe. Wie groß der Spannungsanstieg genau ist, wird entsprechend von den Materialeigenschaften bestimmt. Solange sich beide Werkstoffe im elastischen Bereich befinden, spielt es für den Spannungszustand keine Rolle, ob ein höheres Übermaß gefertigt wird oder ob die Pressung ausgehend von einem geringeren gefertigten Übermaß durch Erwärmung erhöht wird. Dieselbe Übermaßdifferenz führt zu derselben Fugendruckerhöhung und dem somit gesteigerten übertragbaren Drehmoment. Zum anderen hängt der Spannungszustand von der Geometrie der Fügepartner ab. An Wellen und Naben, die bündig enden, treten (an dieser Stelle) keine Spannungsüberhöhungen auf. Auch Wellenabsätze und Ausrundungen kommen einem konstanten Spannungsverlauf entgegen $[4,7]$.

Simulative Untersuchungen haben gezeigt, dass das Verfahren aus [5] vergleichsweise einfach modifiziert werden kann, um zu einem gleichmäßigen Fugendruck zu gelan- gen. Durch geschickte Bestimmung der Reibkräfte lässt sich dieses Ziel erreichen. Der Ablauf ist auch in Abb. 5 dargestellt. Analog zu [5] wird aufbauend auf der Kenntnis des Nennübermaßes (1) mit einer thermomechanischen Fügesimulation begonnen. Dafür wird jetzt das Nennübermaß bei Betriebstemperatur verwendet. Im Anschluss an den Fügevorgang wird die Erwärmung beider Bauteile auf Betriebstemperatur (2) simuliert. Insgesamt betrachtet treten die größten Reibkräfte in diesem Fall ganz am Ende der Simulation (beide Bauteile vollständig gefügt und erwärmt) auf. Wie bisher werden diese Reibkräfte zur Berechnung der benötigten Wellenkontur (4) verwendet. Bei den Einzelsimulationen von Welle und Nabe handelt es sich nach wie vor um rein strukturmechanische Rechnungen (3). Deshalb ist zu beachten, dass sie mit dem korrespondierenden Fugendruck bei Raumtemperatur durchgeführt werden müssen.

Mittels einer Kontrollrechnung kann schließlich der erreichte Verlauf des Fugendrucks überprüft werden. Dazu wird erneut der Fügevorgang mit anschließender Erwärmung simuliert, dieses Mal jedoch unter Verwendung einer Welle, auf die die zuvor berechnete Kontur aufgebracht ist. In Abb. 6 ist das Endergebnis in Prozent der zulässigen Spannung dargestellt. Es zeigt sich, dass der Fugendruck im größten Bereich gleichmäßig verteilt ist, wenn er auch ein wenig über dem Soll-Fugendruck liegt. Zum Nabenende hin (rechts) fällt er ein wenig ab. Zum Wellenende hin (links) bleibt eine geringe Überhöhung bestehen. Interessant ist deshalb zunächst vor allem der Vergleich mit den bisherigen Ergebnissen. Die Spannungsspitze liegt nur noch bei der 1,4-fachen Höhe des Sollwertes. In der Pressverbandmitte liegt der Fugendruck etwa 9\% über dem Soll. Zusätzlich sinkt der Fugendruck zum Nabenende hin nicht mehr so stark ab. Hinsichtlich Reibdauerbeanspruchung ist 


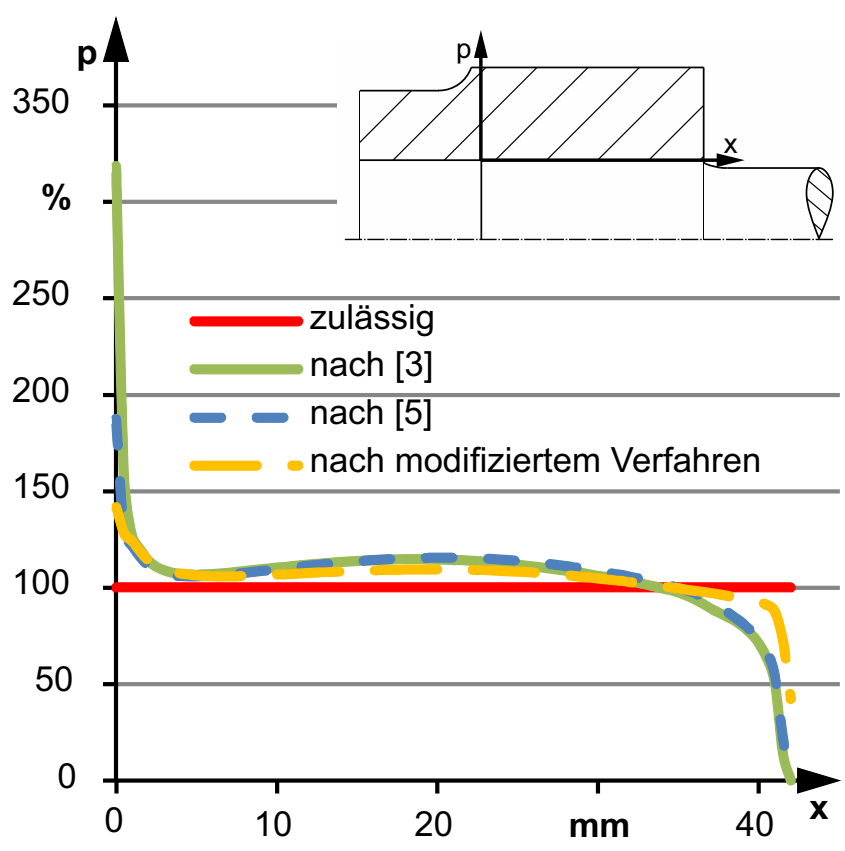

Abb. 6 Fugendruck in Prozent der zulässigen Spannung nach Fügevorgang und Erwärmung auf Betriebstemperatur im modifizierten Verfahren

dieses Verhalten als Vorteil zu werten, weil der höhere Fugendruck eine stärkere Anpressung der Fügepartner aneinander bedeutet und damit zu einer geringeren Reibarbeit im Fügespalt führt. Gerade für dynamische Anwendungen wird auf diese Weise einer Oberflächenzerrüttung und infolge dessen einem Versagen der Welle durch Reibkorrosion vorgebeugt.

\section{Weitere Aspekte der Auslegung und Gestaltung}

\subsection{Optimierung der Ränder des Fügebereichs}

Grundsätzlich ist das vorgestellte Verfahren unabhängig von der Wellen- und Nabengeometrie. Doch wie bereits kurz angesprochen, können sich verschiedene geometrische Kombinationen mehr oder weniger günstig auf den Spannungsverlauf in den Randbereichen auswirken. Das in den vorangegangenen Kapiteln betrachtete Geometriebeispiel zeigt beispielsweise selbst unter Anwendung des modifizierten Verfahrens an der Stelle Nabenende/WellenKorbbogen einen Abfall auf weniger als die Hälfte des Soll-Fugendrucks (Abb. 6). An der Stelle Wellenende/ Nabenüberstand hingegen bildet sich weiterhin eine Spannungsspitze aus.

Die Ursache von Spannungsspitzen an den Rändern der Pressverbindung sind geometriebedingte Steifigkeitssprünge. Enden Welle und Nabe bündig, so entstehen keine un- beabsichtigten Überhöhungen. Endet eines der Bauteile abrupt, während das andere übersteht, so mangelt es an dem kürzeren Teil an stützendem Material. Das Ergebnis ist, dass der Fugendruck nach oben schnellt. Das hier vorgestellte Verfahren löst das Problem durch die Addition der Verformungen von Welle und Nabe. Abnehmende Steifigkeit zum Bauteilrand hin wird so durch sich ändernde Verformungen gleich bei der Konturerstellung mitberücksichtigt. Weitere Untersuchungen haben gezeigt, dass dieses Vorgehen insbesondere bei einem Überstehen der Welle am Nabenende grundsätzlich sehr gute Ergebnisse liefert. Das gilt sowohl, wenn die Nabe an einem Wellenbund anliegt als auch wenn der Wellendurchmesser nach Nabenende reduziert wird. Zu sehen ist dies auch am Ergebnis des Beispiels aus Abschn. 3 (Abb. 6, rechte Seite des Pressverbands).

Ungünstiger wirkt sich ein Überstehen der Nabe über das Wellenende aus. Wie in Abb. 6 zu sehen ist, bildet sich im Beispiel des vorangegangenen Kapitels auf der linken Seite des Pressverbands nach wie vor eine Spannungsspitze aus (wenn auch bereits in der Höhe reduziert). Die Ursache hierfür ist in Abb. 7 erkennbar. Dort ist ein vergrößer-

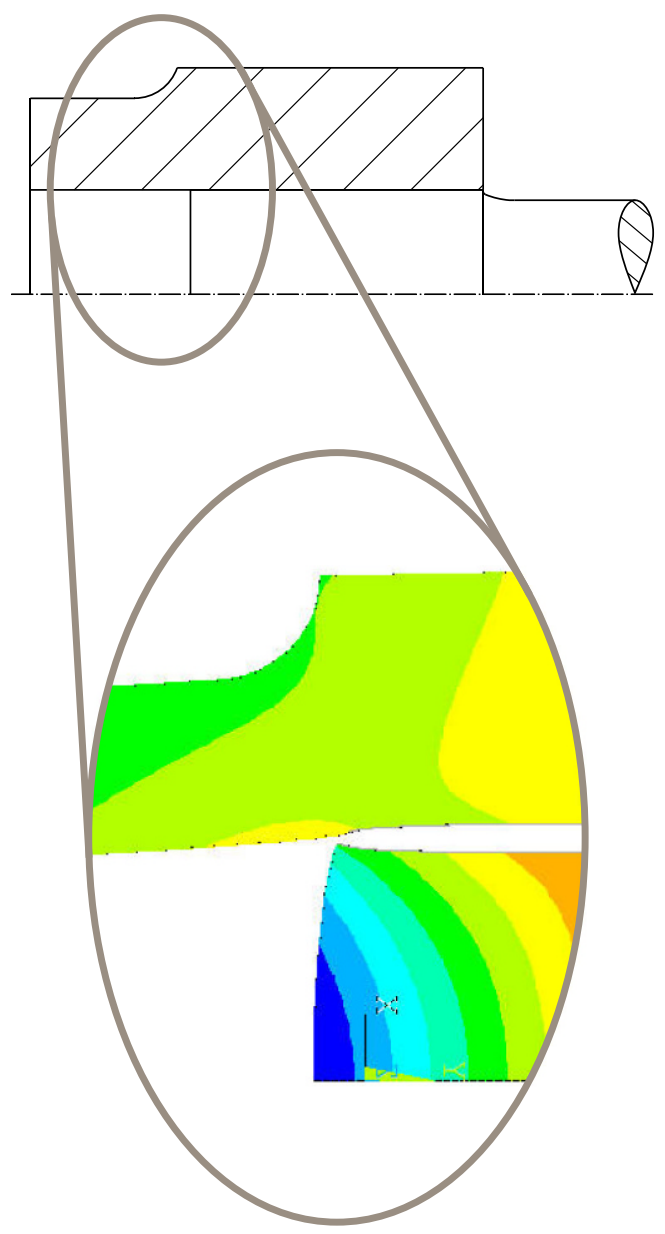

Abb. 7 Überhöhte Darstellung der Verformungen des gefügten Pressverbands bei Betriebstemperatur im Bereich der Spannungsspitze 


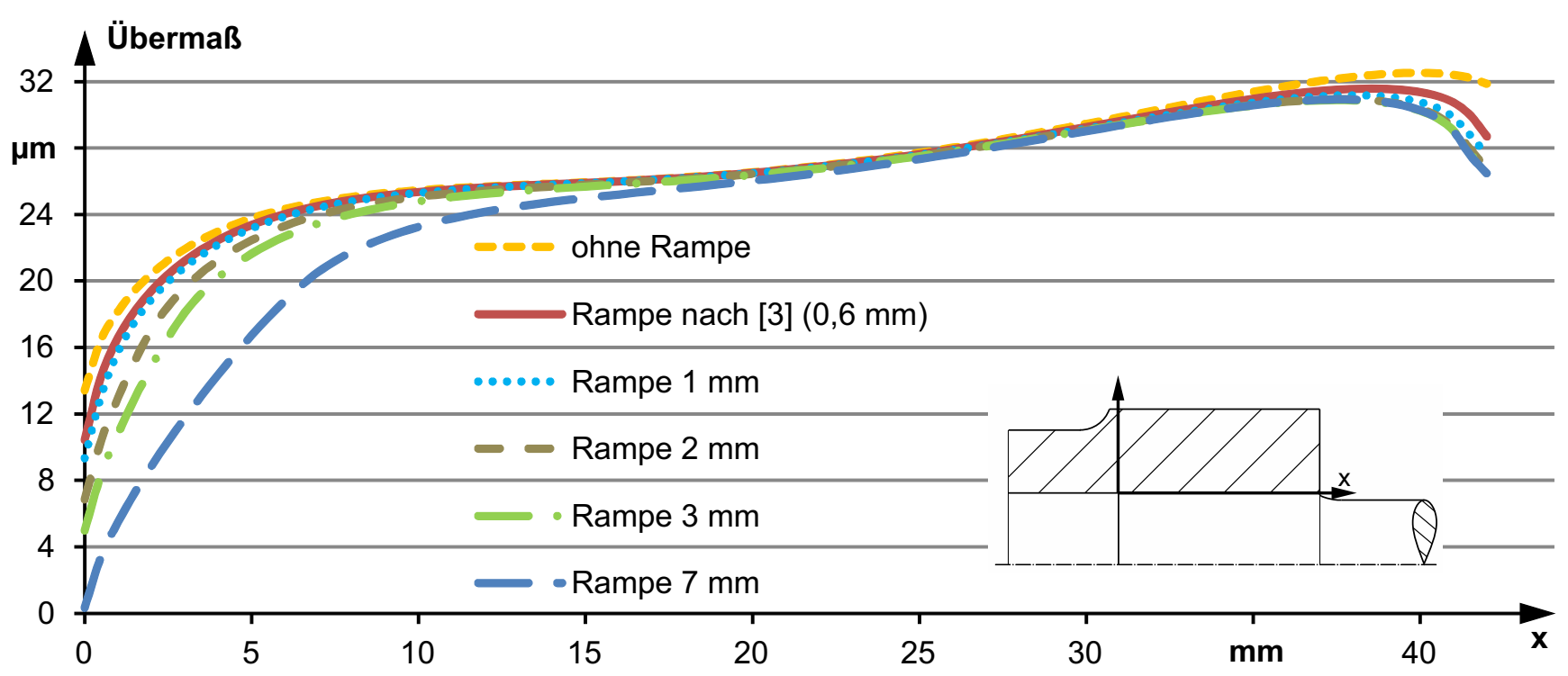

Abb. 8 Wellenkonturen bei unterschiedlich breiten Rampen

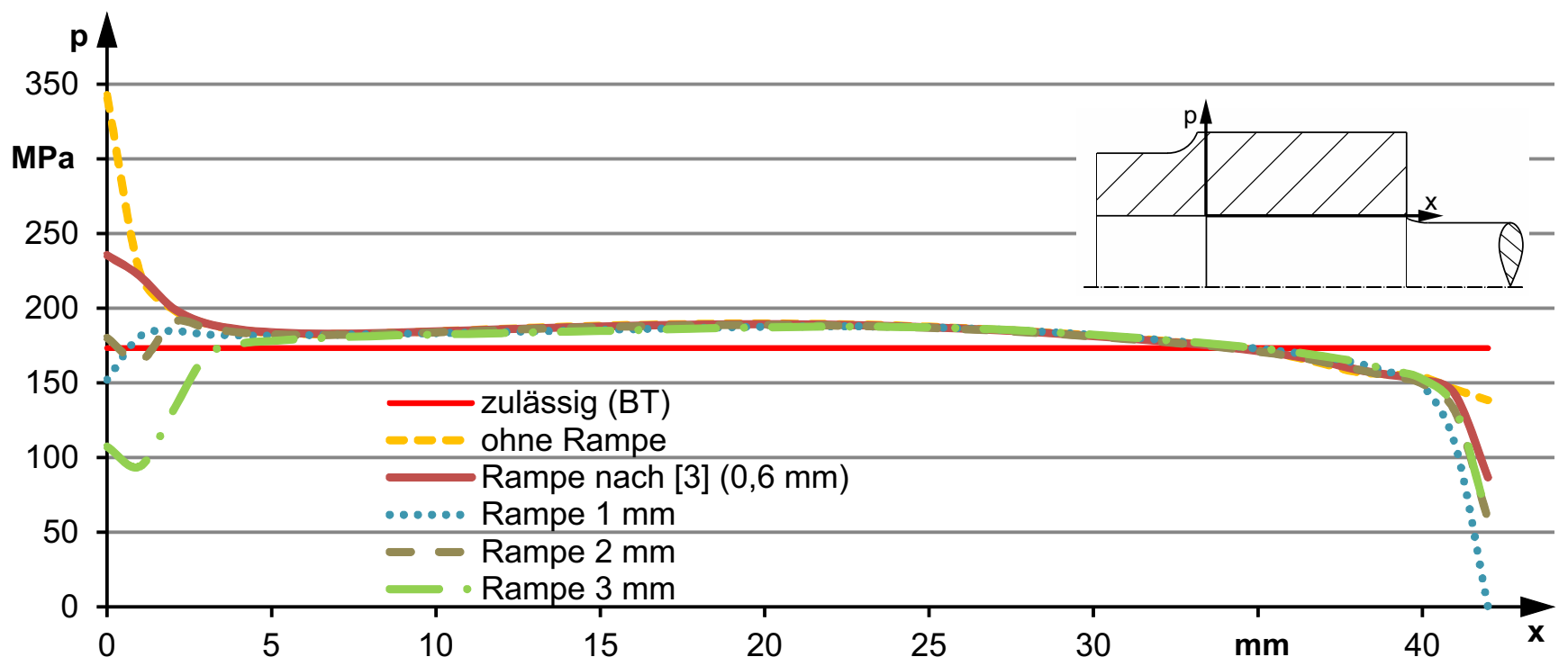

Abb. 9 Einfluss unterschiedlich breiter Rampen auf den entstehenden Fugendruck bei Betriebstemperatur

ter Ausschnitt des Wellenendes aus einer Finite-ElementeSimulation dargestellt. Die Farbverteilung bildet die Verschiebung in axialer Richtung ab. Durch die Überhöhung zeigt sich, dass sich das Welleninnere weiter in Längsrichtung verschiebt als die Fügefläche. Dieses Verhalten lässt sich damit begründen, dass sich die Welle durch die radiale Pressung in der Nabe ,,verhakt" und sich dann trotz Temperaturerhöhung nicht weiter axial ausdehnen kann. Die Wärmedehnung bewirkt dann einen Anstieg des Fugendrucks. Zwangsläufig verstärkt sich dieses Problem bei höherem Übermaß bzw. höherer Betriebstemperatur, was einander entspricht.

Soll die Außengeometrie nicht verändert werden, lässt sich die Spannungsverteilung in den Randbereichen der
Pressverbindung auch durch die Art und Weise beeinflussen, wie der Fugendruck in den getrennten Simulationen von Welle und Nabe aufgebracht wird. Seit [3] wurde der Fugendruck stets mit einer kleinen Rampe aufgebracht, innerhalb derer der Fugendruck von Null auf den Maximalwert ansteigt bzw. am Ende wieder abfällt (vgl. Abb. 1). Tatsächlich beeinflusst die Breite der Rampe direkt die Wellenkontur. Dies ist in Abb. 8 zu sehen, wo die Rampengröße schrittweise von Null über die Rampe von [3] bis hin zu mehreren Millimetern erhöht wurde.

Bei überstehenden Wellengeometrien kann der Fugendruck prinzipiell ohne Rampe aufgebracht werden. Wie in Abb. 9 rechts $\mathrm{zu}$ sehen ist, ergibt sich dadurch sogar eine bessere Annäherung an den Sollwert. Bei überstehenden 
Nabengeometrien lässt sich sagen, dass eine Vergrößerung der Rampe generell zu sinkenden Spannungen im Randbereich führt (vgl. Abb. 9 links). Detaillierte Untersuchungen bzgl. des Zusammenhangs zwischen geometrischen Randbedingungen (Fügedurchmesser etc.), Rampengröße und resultierenden Spannungen sind Gegenstand weiterführender Untersuchungen. Jedoch scheint es sich hierbei um ein recht einfaches Hilfsmittel zu handeln. Wenn nötig kann die optimale Geometrie auf diesem Weg iterativ ermittelt werden.

\subsection{Notwendigkeit von Sicherheitsfaktoren}

Innerhalb des Auslegeverfahrens sind keine Sicherheitsfaktoren explizit festgelegt. Je nachdem, wie genau die Höhe der zulässigen Spannung bekannt ist, kann es notwendig sein, einen zusätzlichen Sicherheitsfaktor einzuführen. Auch mit den Optimierungen aus Abschn. 4.1 ermöglicht es bislang kein Auslegeverfahren für den hybriden Querpressverband, den Soll-Fugendruck auf der gesamten Fügelänge exakt einzuhalten. Die auftretenden Abweichungen sind so gering, dass der sich einstellende Spannungszustand bei konservativer Bestimmung der zulässigen Spannung ertragen werden kann. Dies lässt sich mit Versuchen aus verschiedenen DFG-Projekten bestätigen [8-10]. Sollten jedoch Unsicherheiten hinsichtlich der ertragbaren Spannung bestehen, empfiehlt es sich, nicht an die Grenze des rechnerisch möglichen Übermaßes zu gehen bzw. dieses um eine gewisses Maß zu reduzieren. Die Abweichungen in der Mitte des Fügebereichs liegen unter $10 \%$ und die Belastung der Keramik erfolgt rein elastisch. Entsprechend ist eine Reduzierung des rechnerisch möglichen Übermaßes mit einem Faktor $\mathrm{S} \geq 1,1$ zweckmäßig.

\subsection{Umgang mit gestufter Nabenaußengeometrie}

Allgemein hängt bei Pressverbindungen das benötigte Übermaß vom Durchmesserverhältnis der Nabe ab. Je dünnwandiger die Nabe ist, desto größer ist nach [4] das benötigte Übermaß. Für gestufte Naben könnte das theoretisch bedeuten, dass das Übermaß über der Pressfugenlänge variieren muss. Im Rahmen des simulationsbasierten Auslegeverfahrens für hybride Querpressverbände haben Untersuchungen gezeigt, dass die besten Ergebnisse erzielt werden, wenn über die gesamte Länge vom größten benötigten Übermaß ausgegangen wird. Dadurch kommt es zu höheren Reibkräften, die allgemein zu einer Absenkung der Kontur in der Mitte des Fügebereichs führen. Gerade bei Naben, deren maximaler Außendurchmesser in etwa mittig liegt, wird auf diese Weise der ohnehin leicht erhöhte Fugendruck in diesem Bereich nicht zusätzlich verstärkt.

\subsection{Weitere Besonderheiten bei erhöhten Betriebstemperaturen}

In allen Anwendungsfällen, in denen das benötigte Drehmoment bei erhöhten Temperaturen übertragen werden muss, ist zu beachten, dass das zu fertigende Übermaß kleiner ist als das tatsächlich im Betrieb wirkende Übermaß. Das heißt, dass das volle benötigte Übermaß nach dem Fügen noch nicht vorhanden ist sondern erst bei Betriebstemperatur erreicht wird. Damit kann auch das volle geforderte Drehmoment erst nach Erwärmung von Welle und Nabe auf die Zieltemperatur übertragen werden.

Möglich sind außerdem Fälle, bei denen unter Raumtemperatur noch gar kein Übermaß besteht und die Teile nur lose auf einander gesteckt werden können. Bei solchen Konstellationen müssen geeignete konstruktive Maßnahmen vorgesehen werden, um die Nabe in axialer Richtung präzise zu positionieren. Denkbar ist beispielsweise ein Anschlagbund an der Welle, der ein Verrutschen verhindert.

Zusätzlich muss im Voraus abgeschätzt werden, wie das Anlaufverhalten der Verbindung aussehen soll. Wird die Welle angetrieben bevor der Pressverband fest sitzt, kommt es zu einer verstärkten Reibbelastung im Fügespalt, die zu Schädigungen führen kann und deshalb sicher unerwünscht ist. Zu empfehlen wäre dann ein Vorwärmen der Verbindung, das zur Ausbildung des Pressverbands führt, bevor ein Drehmoment übertragen werden muss. Zugegebenermaßen handelt es sich dabei eher um eine theoretische als um eine praxisgerechte Möglichkeit. Wird der maximale Beanspruchungszustand noch nicht voll ausgeschöpft, ist allgemein auch der pragmatische Ansatz einer ÜbermaßErhöhung denkbar. Diese kann das Fügen in Raumtemperatur-Umgebung ermöglichen, auch wenn sie für den Betriebszustand nicht nötig wäre.

\section{Zusammenfassung}

Die vorliegende Veröffentlichung befasst sich mit der Auslegung hybrider Querpressverbände für Betriebstemperaturen oberhalb der Raumtemperatur. Bisher vorhandene Ansätze basierten auf rein strukturmechanischen bzw. einer Kombination aus struktur- und thermomechanischen Simulationen zur Berechnung einer Wellenform, die zu einem homogenen Fugendruck in der Keramiknabe führt. Beide Ansätze sind nur für den Betrieb bei Raumtemperatur gültig. Wird die Umgebungstemperatur erhöht, so treten erneut unerwünschte Spannungsspitzen auf, die zum Versagen der Naben führen können. Ein großer Vorteil der Keramik ist jedoch ihre hohe Temperaturbeständigkeit, weshalb der Einsatz hybrider Pressverbindungen naheliegend und wünschenswert ist. Deshalb wurde das thermomechanische 
Verfahren gezielt weiterentwickelt, um einen homogenen Fugendruck auch bei höheren Betriebstemperaturen zu ermöglichen. Die Ergebnisse werden ausführlich dargestellt. Sie zeigen, dass mit dem modifizierten Verfahren bereits sowohl Spannungsspitzen deutlich reduziert werden als auch ein unnötiger Spannungsabfall vermieden wird. AbschlieBend werden Hinweise für die praktische Anwendung bei erhöhten Temperaturen gegeben. Sie umfassen sowohl die Spannungsoptimierung mittels Simulationen als auch ggf. notwendige konstruktive Maßnahmen zur Umsetzung der Verbindung beim Fügen und im Betrieb.

Funding Open Access funding enabled and organized by Projekt DEAL.

Open Access Dieser Artikel wird unter der Creative Commons Namensnennung 4.0 International Lizenz veröffentlicht, welche die Nutzung, Vervielfältigung, Bearbeitung, Verbreitung und Wiedergabe in jeglichem Medium und Format erlaubt, sofern Sie den/die ursprünglichen Autor(en) und die Quelle ordnungsgemäß nennen, einen Link zur Creative Commons Lizenz beifügen und angeben, ob Änderungen vorgenommen wurden.

Die in diesem Artikel enthaltenen Bilder und sonstiges Drittmaterial unterliegen ebenfalls der genannten Creative Commons Lizenz, sofern sich aus der Abbildungslegende nichts anderes ergibt. Sofern das betreffende Material nicht unter der genannten Creative Commons Lizenz steht und die betreffende Handlung nicht nach gesetzlichen Vorschriften erlaubt ist, ist für die oben aufgeführten Weiterverwendungen des Materials die Einwilligung des jeweiligen Rechteinhabers einzuholen.

Weitere Details zur Lizenz entnehmen Sie bitte der Lizenzinformation auf http://creativecommons.org/licenses/by/4.0/deed.de.

\section{Literatur}

1. Heinrich U (2002) Untersuchung einer kraftschlüssigen Verbindung von Keramiklaufrad und Metallwelle in Kleingasturbinen. Dissertation, TU Berlin

2. Nitta I, Nakashizuka K, Hara T (1991) The Fitting Strength between Ceramic and Metal with the Use of a Bimetal Shrink Fitter at Elevated Temperature. Jsme Int J 34(2):249-256

3. Blacha M (2009) Grundlagen zur Berechnung und Gestaltung von Querpressverbänden mit Naben aus monolithischer Keramik. Dissertation, Universität Stuttgart

4. DIN (Hrsg) (2017) DIN 7190-1: Pressverbände - Teil 1: Berechnungsgrundlagen und Gestaltungsregeln für zylindrische Pressverbände. Beuth Verlag, Berlin

5. Krautter M, Binz H (2015) Improvement of the designing method of hybrid interference fits. In: Nafems World Congress 2015. Nafems Ltd, San Diego

6. Munz D, Fett T (2001) Ceramics: Mechanical Properties, Failure Behaviour, Materials Selection. Springer, Berlin

7. Haberhauer H, Bodenstein F (2011) Maschinenelemente: Gestaltung, Berechnung, Anwendung. Springer, Berlin

8. Blacha M, Binz H (2008) Basic numerical and experimental examinations for the component design of a hybrid interference fit using fully ceramic hubs. 2nd International Congress on Ceramics, June 29-July 4. Verona, Italy

9. Binz H, Wagner M (2010) Hybride Querpressverbände mit Naben aus monolithischer Keramik unter Torsionsbelastung. Abschlussbericht zum DFG-Projekt BI 746/3. Univ Stuttg

10. Binz H, Schmauder S (2016) Untersuchungen zu Eigenspannungen, Mikrostruktur und Schädigung im Fügebereich von hybriden Querpressverbänden mit keramischer Nabe. Abschlussbericht zum DFG-Projekt BI 746/13 und SCHM 746/127. Univ Stuttg 